Review article

\title{
Current and future role of immobilized enzymes in medical field
}

\author{
Mohammad Rafiq Khan \\ Department of Environmental Studies, Lahore School of Economics, Lahore, Pakistan
}

E-mail: drrafiq@lahoreschool.edu.pk; Phone: +92-4236560954; +92-3219991005

\begin{abstract}
Article history
Received : December 27, 2020

Accepted : January 07, 2021

\section{Keywords}

Anti-inflammatory agents Artificially immobilized enzymes

Immunosensors

Matrix entrapped enzymes
\end{abstract}

\begin{abstract}
Although the history of immobilized enzymes and their applications in different fields are traced back to the second half of the twentieth century, their importance in bioreactors and biosensors highlighted at the turn of the current century is under active consideration in these days for broad-spectrum applications in different medical fields. Thus, this article presents a review of the literature concerning the current and future role of the immobilized enzymes in different medical fields. As the author and his supervised research groups have also been actively involved in research on immobilized enzymes, he has also made some input with a recommendation to revise the current classification of immobilized enzymes basically into "Naturally Immobilized" and "Artificially Immobilized Enzymes", keeping the sub-classification intact. He has also suggested the development of some medical products based on the results of his research groups.
\end{abstract}

\section{INTRODUCTION}

Enzymes are biological catalysts produced by living cells to catalyze cellular reactions essential for the vitality of the organisms. Almost all biochemical reactions in a living body are enzymecatalyzed. The enzymes are finding extensive applications in various fields such as brewing, food production, textile industry, tanning, leather, etc. and are also being used as diagnostic tools and treatments in medicine. Their use is being extended, at present, to many other fields such as agriculture and biochemical research. Enzymes being thermo-labile have presented the problem of the preservation of their life. That is why the scientist all over the world are searching for suitable methods to increase life spans of these biologically important compounds. One of the methods being studied involves their immobilization by their trapping in certain active materials or by binding with specific matrices. The enzymes in the immobilized state are less subject to denaturation and thus can be preserved over time.

Several techniques have been developed to prepare immobilized enzymes because they have exhibited several advantages over enzymes in the solution state (Mohamad et al., 2015). For instance, immobilization leads to ease of recoverability and reusability of the enzymes. Proteolytic enzymes (proteases) refer to various enzymes that hydrolyse and thus breakdown proteins into smaller units such as proteases, peptones, amino acids, etc.

Proteolytic enzymes can be immobilized on different materials and subsequently applied for continuous hydrolysis of protein substrates. Enzymes are usually immobilized by binding them with some matrices filled in chromatographic columns and subsequently passing through them the buffered substrates and collection of products of hydrolysis eluting out of the column. Enzymes immobilized in this way may increase the stability of enzymes to thermal shocks and extremes of $\mathrm{pH}$. The enzymes, after immobilization, undergo certain changes in the spectrum of their activity. Fortunately, many enzymes remain significantly active after their immobilization. Thus immobilization leads to ease of recoverability and reusability of the enzymes.

Proteases refer to various enzymes that breakdown proteins into smaller units that may recombine to yield different proteins essentially required by the organic body while this process of breaking and reforming is in operation continuously in living organisms. The process of breakdown of protein by the enzyme protease into peptides, amino acids, etc. is called proteolysis. Although the observations about the action of proteolytic enzymes on proteins made the discovery of the cleavage of the peptide bond in 
the $19^{\text {th }}$ century, at that time there was no clear sketch of the mode of action of enzymes. Later, when it became known that the amino acids were linked by the peptide bonds in proteins, the experts paid full attention to understand the mode of action of theses enzymes on protein and utilize proteases on a commercial scale. The proteases have also found extensive applications in medicine as cures of diseases such as stomach upset, fistulas, hyperacidity and cramps. They are being extensively used as anti-inflammatory agents. They digest dead cells of skins necrotic tissue and thus act as wound cleaners. Similarly, they are being extensively applied as cleaners of contact lenses.

On the basis of the importance of proteases highlighted above, the proteases have been under extensive research in the past and, even, are currently being intensively studied, all over the world. The countries involved in this activity are China, Australia, Kenya, Hungry, Bangladesh, Japan, Turkey, America, India, Pakistan, etc. China is the leader to utilize plant proteases on a commercial scale. Some significant reports have appeared which highlight the diversity of applications of proteases in Pakistan. Amino acids and other products of proteolysis have extensive applications in different fields. The mixture of amino acids can be transfused with the help of drips into the body of patients so that the supply of these essential nutrients for the synthesis of structural and functional proteins, essentially required for the body, may be ensured. Amino acids may also be separated for use in Chemistry laboratories. Finally, there are many other applications of amino acids.

Considerable work has been carried in Pakistan to hydrolyze casein by proteases immobilized on different materials keeping in view the above-mentioned applications. An attempt was made to develop continuous proteolysis systems consisting of protease immobilized by combining with artificial and naturally occurring materials such as DEAE A-50 cellulose, Amberlite-50, Activated Charcoal, etc. (Khan and Talib, 1986; Nawaz and Khan, 2000).

Later, the work was extended to the study of naturally immobilized proteases of vegetable origin such as Carum copticum, Allium sepa, Nigella sativa (Khan and Bukhari, 2008; Khan et al., 2008). The difficulty encountered was that there was also contained in the cells of the seeds the soluble protease component. Thus, the seeds were investigated for both soluble and immobilized proteases. The results, of course, revealed that major component was the immobilized one while soluble component was a minor one. This difference made a good ground for the study of naturally immobilized enzymes packed in chromatographic columns. Thus the work was extended by the elution of soluble enzyme so that only the cell bond enzyme may react with the substrates.

\section{CLASSIFICATION OF IMMOBILIZED ENZYMES}

The term "immobilized enzymes" was recommended at the first Enzyme Engineering Conference in 1971 (Wingard, 1974). The enzymes were first classified into two groups, viz. Native enzymes and Modified enzymes. Immobilized enzymes belong to the class of modified enzymes and are further divided into two groups, entrapped immobilized enzymes and bound immobilized enzymes.

\section{Entrapped immobilized enzymes}

Entrapped enzymes are those enzymes which can be entrapped in a semi-permeable gel or which can be enclosed in a semi-permeable polymer membrane. For example, glucoamylase is entrapped by polyacrylic acid and invertase by polyvinyl alcohol. Entrapped enzymes are further divided into two sub-classes.

\section{Matrix entrapped enzymes}

Matrix entrapped enzymes are those which are entrapped within the interstitial spaces of crosslinked water-insoluble polymers, i.e. within a gel. For example, alcohol dehydrogenase is entrapped within polyacrylamide and invertase is trapped within polythene glycol polymer.

\section{Microencapsulated enzymes}

These are those enzymes, which are enclosed within the semi-permeable polymer membranes.

\section{Bound immobilized enzymes}

Bound immobilized enzymes like entrapped enzymes are further sub-divided into two classes, viz. adsorbed bound immobilized enzymes and covalently bound immobilized enzymes

\section{Adsorbed bound immobilized enzymes}

The adsorbed immobilized enzymes are those which are immobilized by binding with an adsorbent such as activated charcoal, micro-glass beads, etc.

\section{Covalently bound immobilized enzymes}

The covalently bound immobilized are those which are immobilized by a matrix through a covalent chemical linkage.

\section{APPLICATION OF IMIMOBILIZED ENZYMIES}

Immobilization results into certain changes in the properties of the enzymes, which form the basis of their applications in medical fields and industry and also for the elucidation of chemical structure, 
function, relationship and mechanisms of enzymatic action. The enzyme properties are mainly changed due to two factors, the changes of the enzyme itself and the physical and chemical properties of the carriers used for immobilization.

The important properties that undergo changes to make immobilized enzymes beneficial include substrate specificity, temperature optima, thermostability, $\mathrm{pH}$ optima, $\mathrm{pH}$ stability, stability towards reagents, stability towards proteolytic enzymes, operational stability, increase in shelf life, activation energy, kinetic constants, etc. Most of the changes are favourable for the qualification of immobilized enzymes in different fields. This may be illustrated with the results of a study reported by Markoglou and Wainer (2003). The immobilized enzyme phenylethanolamine $\mathrm{N}$-methyltransferase (PNMT) displayed an increase in thermal stability under the effect of temperature. The increased stability may be due to the reason that immobilization limits the thermal movement of the enzyme at the higher temperatures as a result of which denaturation may take place at higher temperatures. It was suggested that the higher enzymatic thermo-stability may allow faster reaction rate, lower diffusional restrictions, increased stability and greater yields that are adequate qualifications for the applications of immobilized enzymes in industry. Similarly, the $\mathrm{pH}$ stability increase after immobilization may translate into higher yields of products, while the operational stability is desirable because the enzymes should remain intact with least loss of activity during the industrial operations of the production line to have higher product yields. The longer shelf life of the enzymes is also an important requisite to keep the enzyme intact for its long time use in industry. Luckily, the binding of an enzyme with a matrix or its encapsulation helps in imparting long life due to its protection from the environmental shocks of the chemical reagents and physical factors such as heat, light and others.

\section{CURRENT AND FUTURE APPLICATIONS OF IMIMOBILIZED ENZYMMES IN MEDICINE}

The medical applications of immobilized enzymes have been extensively studied and developed (Li and Rosenzweig, 1997; Liu et al., 1997; Magnani et al., 1993; Marazuela et al., 1997; Marazuela and Moreno-Bondi, 1998; Mintzer and Hauptman, 1983; Shen and Cho, 1995; Tamer et al., 2016; Torres-Bacete et al., 2000) and successively reviewed by many experts (Chang, 1977; Ghosh and Parida, 2015; Homaei et al., 2013; Liang et al., 2000; DeLoach and Droleskey, 1993; Garin et al., 1995; Krishnamoorthi et al., 2015; Mohamad et al., 2015). Here, only highlights of most significant studies and salient features of the reviews are described. Enzymes immobilized by entrapping method particularly offer their therapeutic use in case of an enzyme deficiency or metabolic disorders. Liang et al. (2000) described the medical applications of immobilized proteins, enzymes and cells. The researchers used microencapsulated catalase for enzyme replacement in acatalasemic mice (mice exhibiting oral gangrene) due to congenital deficiency of catalase. The report also discussed the use of liposomes for the microencapsulation of enzymes. The liposomes have the advantage of being biodegradable; but, they appear to enhance the immune response to the entrapped protein. Moreover, the multi-enzyme systems enclosed within artificial cells are capable of use for converting ammonia or urea into amino acids. The report also discussed the use of asparaginase and other enzymes in chemotherapy with reference to their use associated with problems related to toxicity, immunogenicity, and duration of action. The report says that "initial attempts at immobilization resulted in the successful use of microencapsulated asparaginase to suppress the growth of lymphosarcoma in mice". Lymphoma (lymphosarcoma) is the third most common cancer diagnosed in dogs. It is a cancer of lymphocytes, a type of blood cells and lymphoid tissues. Lymphosarcoma cell leukaemia forms the basis to refer to three related clinical syndromes: primarily the invasion of peripheral blood by poorlydifferentiated lymphocytic lymphoma, secondly the invasion of blood by other types of lymphoma, including large cell describes a variant of chronic lymphocytic leukaemia, presenting clinically without lymphoma and thirdly the invasion of blood by other types of lymphoma, including large cells, lymphoblastic, and Burkitt's lymphoma (Mintzer and Hauptman, 1983). Because of the importance of cancer treatment, many studies have been carried out using all available immobilization approaches. The immobilized enzymes are used in detoxification because many of the detoxifying functions in the body are carried out by enzymatic reactions. Chang (1988), for instance, has investigated the application of enzymes microcapsules entrapped inside a semi-permeable polymembrane. Later, workers prepared asparaginase microcapsule and attempting to use them for the treatment of leukaemia. They also studied the changes in asparaginase level in blood after administration of asparaginase microcapsule.

Utilization of immobilized enzymes for the treatment of blood outside the body i.e. "Extra Corporeal Shunt System" has also been attempted. Mori et al. (1974) investigated the application of immobilized asparaginase prepared by entrapping in polyacrylamide gel which is mechanistically stronger than the enzyme microcapsule for the therapy of leukaemia by means of an extracorporeal shunt system. This preparation was very stable towards the proteolytic enzymes and Lasparagine in blood was completely decomposed when blood was passed through a column packed with an immobilized enzyme. 
An extracorporeal shunt system is known to have great potential and may be applied for emergency cases. The time required for such a therapy can be controlled easily, and specific detoxicating material used for the system can be prepared readily.

With the background of the importance of immobilized enzymes in the medical field as highlighted above many studies and reviews dealing with the current and future applications in different fields have appeared in literature along with bringing into focus the techniques of immobilization and changes in the properties of enzymes after going through the process of immobilization.

Kilbanov (1983) presented a comprehensive review of the performance of enzymes and whole cells in commercial applications and claimed that it can be dramatically improved by immobilization of the biocatalysts by their covalent linkage to an appropriate matrix or by adsorption on solid supports such as polymeric gels, encapsulation, and cross-linking. The effect of immobilization on enzymatic properties and stability of biocatalysts was also discussed. Applications of immobilized enzymes and cells in the chemical, pharmaceutical, and food industries, in clinical and chemical analyses and medicine, as well as probable future trends in enzyme technology, were brought into focus.

It may be appropriate to point out here that some reviews have a wider spectrum of their coverage of the subject and thus they have rightly not restricted themselves to the field of medicine only. Instead, they have extended to all medical sciences and even too many other scientific fields including industry and most of the life science discipline. Even when extending to medical sciences, many other disciplines aligned to general medicine such as pharmacology, biochemistry, clinical methods, are talked about.

Matosevis et al. (2011) have presented an overview of fundamental principles and applications of immobilized microfluidic enzymatic reactors and told that the utility of immobilized microfluidic enzyme reactors (IMERs) had been demonstrated in a wide variety of fields such as medical, diagnostics and therapy, biosensors, organic synthesis, drug discovery and many others. Of particular interest to the pharmaceutical industry, in their opinion was "the potential for high throughput experimentation afforded by these systems, with a view to combinatorial synthesis for drug discovery applications." Thus they focused on the current status of IMER systems, including immobilization techniques and micro-channel flow generation, with a particular emphasis on applications and prospects because of likely directions and market potential of this field. The researchers also narrated numerous advantages of attaching enzymes to a solid support such as reuse of a single batch of the enzyme, improved stability and durability, the ability to stop the reaction rapidly by removing the product from the reaction solution and the absence of enzyme contamination of the product are some of the attractive features of such systems." They pointed out several issues requiring careful consideration when developing such microsystems, including, but not limited to, surface modifications and exact control of fluid behaviour in microchannels, detection limitations, increased integration, and the reusability of the chips. As reported the IMERs have found many medical applications, including commercial, application as diagnostic tools for point-of-care applications, and, increasingly, as analytical tools in early drug development. Furthermore, peptide mapping and proteomics have employed IMER systems.

Rao (2012), in his review, attempted to summarize some of the developments in the application of collagen as a biomaterial and drug delivery systems. The main applications include collagen for burn/wound cover dressings, osteogenic and bone filling materials, antithrombogenic surfaces and immobilization of therapeutic enzymes. Recently, collagen used as a carrier for drug delivery has attracted many experts all over the world. The use of collagen for various drug delivery systems has also been to review systems inclusive of injectable microspheres based on gelatin, implantable collagen-synthetic polymer hydrogels; interpenetrating networks of collagen and synthetic polymers collagen membranes for ophthalmic delivery. The review also covered the use of collagen-liposomal composites for controlled drug delivery, as well as collagen as controlling membranes for transdermal delivery.

The focus at the turn of the century was on the applications on the use of immobilized enzymes as biosensors in bioreactors to control the concentration of analytes in the bioreactors in medical sciences. One of the important confusions encountered in this inquiry was that the experts described applications in the field of current medicine instead of medical sciences, The point being presented was on a broad basis and that initiates understanding of what were the "Immobilized Enzymes", what were their characteristics in which they differed from the normal or free enzymes and their applications in all inter-related medical sciences such as diagnostic methodology, pharmacology, pharmaceutical industry, food and nutrition and general medicine with special reference to the medical treatments and drug development.

In general, most biological reaction systems could be classified into two main groups: suspension systems and immobilization systems. Stirred tank, air-lift, and bubble column bioreactors were mainly for suspension cultures, while membrane, packed bed, and fluidized bed bioreactors are mainly for cultivating attached cells 
or immobilized enzymatic reactions. The bioreactor could provide optimum conditions (e.g., temperature, $\mathrm{pH}$, oxygen transfer, mixing, and substrate concentration), in addition to its basic function of containment. Bioreactors were narrated to play an important role in many industries such as fermentation, food, pharmaceuticals and waste water treatment. Bioreactor engineering has been experiencing rapid progress. In recent years, micro-bioreactors have received great attention and are receiving even more due for their perspective use in the pharmaceutical industry. With the tremendous progress in functional genomics, metabolic engineering, and systems biology, there is a great potential for a single cell working as a super bioreactor. The high-level expression and efficient recovery of recombinant proteins are two main critical factors that determine the use of transgenic plants as natural bioreactors.

As the focus is mainly on the use of immobilized enzymes as biosensors and bioreactors in the pharmaceutical industry that manufactures drugs for the medical treatments, let us try to understand what these are? The bioreactor, as defined by Liang et al. (2020), is a vessel that carries out a biological reaction and is used to culture aerobic cells for conducting cellular or enzymatic immobilization. The cell culturing is the most commonly applied process in biopharmaceutical development, bioprocessing and biomanufacturing in the pharmaceutical industry. Most biopharmaceutical products are developed from large, complex protein molecules grown in well- defined and highly controlled liquid environment, inside the bioreactors.

The performance of a bioreactor depends largely on biosensors which are the devices used to detect the presence or concentration of a biological analyte such as a biomolecule, biological structure or microorganism. The biosensor consists of three parts viz. a component that recognizes the analyte and produces a signal, a signal transducer, and a reader device. A biosensor is an analytical device, applied for the detection of a chemical substance that combines a biological component with a physico-chemical detector (Glaxier and McCurley, 1995; Guerrieri et al., 1997). Different biosensors available in the market are as follows.

\section{Electrochemical biosensors \\ 2. Immunosensors \\ 3. Magnetic biosensors \\ 4. Thermometric biosensors \\ 5. Acoustic biosensors \\ 6. Optical biosensors}

Hill et al. (1997) prepared liposomes from phospholipid mixtures of dipalmitoylphosphatidylcholine (DPPC) and phosphatidylinositol (PI), encapsulating the enzymes glucose oxidase and glucose oxidase in combination with horseradish peroxidase by both extrusion (VET) and reverse- phase evaporation (REV). The optimum level of PI in DPPC/PI liposomes for targeting to biofilms of the oral bacterium Streptococcus gordonii has been established. They characterized liposomes in terms of the content and activity of the encapsulated enzymes and measured the antibacterial activity of reactive liposomes due to hydrogen peroxide and oxyacid's in presence of the substrates glucose and iodide ions, after targeting the biofilms, were measured as a function of liposome-biofilm incubation time and as incubation time with the substrates. Bacterial inhibition increased with both liposome-biofilm and substrate-biofilm incubation time and with the extent of enzyme encapsulation. The reactive liposomes also displayed antibacterial activity in presence of saliva. The reactive liposomes exhibited potential value in the context of oral hygiene.

Homaei et al. (1997) developed an on-line electrochemical sensor to measure acetylcholine (ACh) continuously. The sensor comprised of a small volume prereactor containing immobilized choline oxidase (ChOx) and catalase (or horseradish peroxidase, HRP), a small volume enzymatic reactor containing immobilized acetylcholine esterase ( $\mathrm{AChE}$ ) and $\mathrm{ChOx}$ and a glassy carbon electrode modified with Os-polyvinyl pyridine-HRP. The sensor could measure ACh selectively even when the choline (Ch) concentration was more than 1000 times that of ACh when ChOx and catalase were immobilized in the prereactor. The sensor showed a wide linear relationship from $5 \mathrm{nM}$ to $1 \mu \mathrm{M}$ and a detection limit of $5 \mathrm{nM}(\mathrm{S} / \mathrm{N}=2)$, which is comparable to the ACh detection limit achieved by liquid chromatography/ electrochemistry. It also showed high selectivity against other neurotransmitters and interferents. The properties of the sensor combined with microdialysis (MD) probe were also studied by measuring a solution containing a much higher concentration of $\mathrm{Ch}$ than $\mathrm{ACh}$.

$\mathrm{Li}$ and Rosenzweig (1997) fabricated a miniaturized fibre-optic biosensor for rapid analysis of bilirubin. Both 1,10-phenanthroline ruthenium chloride, $\left[\mathrm{Ru}(\mathrm{dpp})_{3}\right]$ and bilirubin oxidase were physically immobilized in an acrylamide polymer matrix that was attached covalently to a silanized optical fibre tip surface via controlled photopolymerization. Leaching of the ruthenium dye was minimized by the optimization of the ratio between the acrylamide monomer and the cross-linker ( $N, N$-methylene bisacrylamide). An $8 \%$ isobutyl alcohol in a phosphate buffer at $\mathrm{pH}$ 6 was used to store the fibre optic biosensor. This solution maintained the enzyme activity and the sensor response and minimized the leaking of bilirubin oxidase from the polymer matrix. The detection limit of the sensor was $1 \times 10^{-7} \mathrm{M}$ and its linear dynamic range was between $1 \times 10^{-7}$ and $3 \times 10^{-4} \mathrm{M}$. The response time of the sensor indicated a 50-fold improvement over existing bilirubin sensing devices. 
De Keijzer et al. (1999) evaluated the analytical performance of a biosensor for the measurement of lactate in whole blood samples and compared the results of patients' samples with a spectrophotometric enzymatic method. For the comparison of these methods, human blood samples were split and measured. The results indicated that the combination of analytical performance, easy handling, rapid analysis, and measurement in whole blood makes the biosensor suitable for lactate STAT-analyses."

Lee and Huh (1999) investigated conditions for immobilization of L-amino acid oxidase (LAAO) on a preactivated nylon membrane, and to develop an enzyme sensor which can detect ammonia. LAAO was immobilized on a preactivated nylon membrane. Optimal immobilization conditions were $0.3 \%$ glutaraldehyde, $1 \mathrm{mg}$ bovine serum albumin, and 1.6 units of the enzyme with $2 \mathrm{~h}$ coupling time. The enzyme membrane and an ammonium selective non-acting membrane were attached to an ammonia electrode to fabricate an L-amino acid sensor. L-amino acids were enzymatically degraded by immobilized LAAO, and the ammonia generated was measured by a potentiometric electrode at $\mathrm{pH} 8.5$ and $45 \mathrm{C}$. The sensor showed high relative activities for L-amino acids, and the responses of the sensor for phenylalanine and isoleucine were linear to $10 \mathrm{mM}$ with a detection limit of $0.05 \mathrm{mM}$. The L-amino acid sensor was applied for monitoring increases in amino acids levels, expressed as L-isoleucine equivalents, during yeast autolysis. Determination of L-amino acids was complete in $3 \mathrm{~min}$. Moreover, the activity of the enzymatic membrane was stable for at least 260 essays and did not noticeably decline for 2 months.

The use of immobilized enzymes in medicine can be divided into two major categories viz. biosensors and bioreactors. Liang et al. (2000) gave a brief overview of the evolution of the biosensor and bioreactor technology pertaining to existing applications of immobilized enzymes, researchers' problems and possible future developments. As compared to free enzymes in solution, immobilized enzymes are more robust and more resistant to environmental changes. More importantly, the heterogeneity of the immobilized enzyme systems allows easy recovery of both enzymes and products, multiple re-uses of enzymes, and continuous operation of enzymatic processes (Homaei et al., 2013).

Babin and Sova (2014) preliminarily developed a fibre optic bilirubin sensor where an unclad sensing portion was used to provide evanescent wave interaction of the transmitted light with the chemical environment. By using a wavelength corresponding to a bilirubin absorption peak, the Beer-Lambert Law could be applied to relate the concentration of bilirubin surrounding the sensing portion to the amount of absorbed light. Initial testing in vitro suggested that the sensor response is consistent with the results of bulk absorption measurements as well as the Beer-Lambert Law. In addition, it is found that conjugated and unconjugated bilirubin has different peak absorption wavelengths, so that two optical frequencies may potentially be used to measure both types of bilirubin. Future development of this device could provide a means of real-time, pointof-care monitoring of intravenous bilirubin in critical care neonates with hyperbilirubinemia.

\section{IMIMOBILIZED ENZYMES RESEARCH DONE BY THE AUTHOR AND HIS GROUP}

A number of research groups composed of master level graduate research students remained associated with the author and many out them worked on the plant and microbial enzymes in Biosection of Government College Lahore (GCL) now Government College University (GCU) while some had a chance to work on immobilized enzymes. The author later continued this exercise while he headed the Chemistry Department Government College of Science Lahore. Initially, the work was undertaken on artificially immobilized enzymes and some good results were obtained. The spectrum of activity was later extended to the cellbound enzymes that were conceived to be naturally immobilized. The concept of natural immobilization was thus introduced by the working groups at their own level because this term was not found then in literature. The seeds such as Carum copticum, Nigella sativa and Allium sepa were made the subjects of inquiry due to their traditionally well-known digestion characteristics currently thought to be due to cell-bound proteases. The results of the inquiry are reported below with the discussion of prospective applications as concluding wind up.

\section{Artificially immobilized proteases}

Khan and Talib (1986) attempted the immobilization of the protease of Calotropis procera with an anion exchanger Amberlite-CG 50 packed in a chromatographic column. The plant was the choice due to its wide choice as a traditional, medicinal plant. The amount of the enzyme immobilized was found to be $23.5 \%$. Thus, a continuous proteolytic system was set up using citrate-phosphate buffer $\mathrm{pH}$ 7.0. To do so amberlite-C50 was packed and through it the extract of Calotrpis procera was run for binding to the matrix and unbound soluble protease was eluted out and assayed. The matrix in the column was washed by running in the buffer till no more soluble protease eluted out of the column. After that $1 \%$ casein substrate in the same buffer was run through the system and the products of proteolysis were assayed as an index to the extent of proteolysis. The system was found significantly active for proteolysis. The life span of the enzyme 
was found to be effective for about 3 weeks. Similar system development was tried using activated charcoal as an adsorbent binder but this time a Buchner funnel was substituted for a chromatographic column to make it cost-effective. Another change made here was that instead of running for many days, the system was just operated for the full day and all the fractions from the funnel were bulked and assayed. The results showed that this system worked well for the investigation of three weeks. It was even active after this period but work was terminated due to time constraint.

Nawaz and Khan (2000) immobilized protease of Eurphobia royleana by its ionic binding with DEAE-A50 cellulose packed in a chromatographic column. The amount of enzyme immobilized was found to be $31 \%$. Thus a continuous proteolytic system was set up that successfully hydrolyzed buffered casein substrate passed through the column. The extent of proteolysis was followed by assay of the photolytic products eluted from the bottom of the column. The life span of the immobilization was 22 days endorsing the inference that the proteases can be immobilized over a significant time to preserve them for a certain period. A continuous proteolytic system has been set up for binding the protease of Carica papaya with DEAE-A50 in a chromatographic column tested by running through the column casein substrate. Here, the result that makes the difference is that its binding was $67 \%$ while the life span was 30 days higher than our previous exercises. A similar exercise has also been done to binding the protease of Carica papaya with Amberlite CG-400 and found that the enzyme binding by $54 \%$ and life span of the immobilized enzyme system was recorded to be $27 \%$ which indicated that DEAE-A 50 was a better binder and life preserver.

\section{Naturally immobilized proteases}

The exercise was continued in higher studies leading to a $\mathrm{PhD}$ degree and it leads to the exploration of new vistas such as naturally immobilized proteases of well-known medicinal seeds: Carum copticum, Allium sepa and Nigella sativa. Thus the primary work was continued on the pattern described above. It was followed by the work on naturally bound plant proteases. These are described below in the same sequence.

It was planned to investigate many seeds that carry medicinal value due to the reason that they contain proteases sticking to the cells in Free State and thus can be dissolved out. The examples of well-known plant seeds may be Carum copticum (Ajwain), Allium sepa (piaz), Nigella sativa (Kalongi) and many others. These are mostly used by the oriental physicians as digestive aids to correct stomach disorders. Thus, the first experiment carried out in this context was the inquiry of Carum copticum (Khan et al., 2008). Its seeds were packed in a chromatographic column that was subsequently washed with citrate-phosphate buffer $\mathrm{pH}$ 7.0. The elution was carried out till all the soluble protease present in the sample was washed out and naturally bound protease immobilized due to binding with the cells remained intact in the column. The buffered substrate (Casein) was applied to the column and column was run collecting five $\mathrm{ml}$ fractions assayed for the hydrolyzed protein products. The elution diagram exhibited its peak that was far higher as compared to all peaks exhibited in other experiments including the peaks displayed by proteolysis of casein by both artificially immobilized and naturally immobilized enzymes describe below. The column life was found to be very long.

A similar exercise was carried out for developing a continuous proteolysis system by packing Nigella sativa seeds in a chromatic column with the subsequent running of the column by application of casein substrate under similar conditions. The extent of proteolysis was significantly high that went on increasing over time. The life span of the cell-bound protease of Nigella sativa was found to be 39 days. The spectrum of inquiry was extended to Allium sepa and to set up the continuous proteolytic system similarly under the same conditions. The system operated successfully for proteolysis.

\section{DISCUSSION}

The author's groups extensively worked on enzymes, immobilized enzymes and cells and reported highly significant results that could be exploited commercially for development of medical drugs and immobilized systems for use in the medical field but they could not because the environment in the institutions of higher learning is more social and less scientific in the applied context. For example, a resolution passed by the audience of a debate in Bukhari Auditorium of Government College Lahore (GCU) was accepted all over the World during the period the research groups worked on different problems. Thus, the results those were highly significant in applied context remained unnoticed by the commerce and industry but they were assigned a high value in the academic context. The groups did not even think in terms of their commercial exploitation.

After having a comprehensive survey of literature in the field for writing the current article, the author felt serious about the losses of his groups. What he feels today is expressed below in the following paragraphs.

The author now disagrees with the classification of immobilized enzymes by Enzyme Engineering Conference (1971) because his groups have worked on cell-bound proteases of medicinal seeds and have given them the name of "Naturally Immobilized Enzymes". Thus, the author 
proposes that the enzymes should be classified into "Naturally Immobilized Enzymes" and "Artificially Immobilized Enzymes". The later should be further sub-classified as done in 1971. That sounds logical and thus acceptable to the author and his associates.

The author and his associates appreciate without any reservation the spectrum of applications of immobilized enzymes and breaking the news that the spectrum being broad, the field of this form of enzymes deserves close attention because it will open new vistas for broadening the spectrum of application for the benefit of future generations. This encourages the author and his associates to give some suggestion based on the results of their research particularly on naturally immobilized enzymes on the basis that whatsoever is natural is safer to use as medical treatment.

1. The artificially immobilized enzymes after binding with the matrices or encapsulated in gels, etc. may be removed from the columns, etc., freeze-dried, powdered and bottled for keeping them intact over a long life.

2. The seeds bearing immobilized enzymes may be packed in the columns in their natural form to develop systems that may operate continuously to hydrolyze proteins to prepare amino acids that may be used to fill up drips for transfusion into the blood of the patients suffering from functional protein-deficient diseases or make up their nutritional deficiency of these or other proteolytic products when they are forbidden for taking any food by mouth.

The immobilized proteases may be used as components of the dialyzers for removal of toxins from the blood before its retuning to the body of the patients with partial kidney failure undergoing dialysis for prolonging their life.

\section{CONFLICTS OF INTEREST}

The author declares no conflicts of interest.

\section{DECLARATION}

The contents of this paper are published after receiving a signed copyright agreement from the corresponding author declaring that the contents of this paper are original. In case of any dispute related to the originality of the contents, editors, reviewers and publisher will remain neutral.

\section{REFERENCES}

Babin SM, Sova RM (2014). Preliminary Development of a Fiber Optic Sensor for Measuring Bilirubin. Analytical Chemistry Insights, 9, 59-65.

Chang TMS (1977). Biomedical Applications of Immobilized Enzymes and Proteins, Vol. 1. Plenum Press, New York.

Chang TMS (1988). Medical applications of immobilized proteins, enzymes, and cells. Methods in Enzymology, $137,444-457$.
De Keijzer MH, Brandts RW, Brans PG (1999). Evaluation of a biosensor for the measurement of lactate in whole blood. Clinical Biochemistry, 32, 109-112.

DeLoach JR, Droleskey RE (1993). Endocytosis during the preparation of mouse and human carrier erythrocytes. Biotechnology and Applied Biochemistry, 18(1), 83-92.

Garin M, Rossi L, Luque J, Magnani M (1995). Lactate catabolism by enzyme-loaded red blood cells. Biotechnology and Applied Biochemistry, 22, 295-303.

Ghosh D, Parida P (2015). Modern Applications of Plant Biotechnology. International Journal of Pharmacology and Pharmacological Sciences, 2(6), 5-10.

Glaxier SA, McCurley MF (1995). Biosensor applications for bioprocess monitoring and drug analysis. Biopharmacology, 8, 38-48.

Guerrieri A, Debenedetto GE, Palmisanso F, Zambonin F, Zambonin PG (1997). A fiber optic sensor for rapid analysis of bilirubin in serum, Images. Analytica Chimica Acta, 353, 263-273.

Hill KJ, Kaszuba M, Creeth JE, Jones MN (1997). Reactive liposomes encapsulating a glucose oxidaseperoxidase system with antibacterial activity. Biochimica et Biophysica Acta, 1326, 37-46.

Homaei AA, Sariri R, Vianello F, Stevanato FR (2013). Enzyme immobilization: an update. Journal of Chemical Biology, 6(4), 185-205.

Homaei T, Torimitsu K, Yamamoto K, Niwa O (1997). On-line flow sensor for measuring acetylcholine combined with microdialysis sampling probe. Electroanalysis, 9, 912-916.

Khan MR, Bukhari H (2008). Immobilization of the protease of Carica papaya on Activated Charcoal. Asian Journal of Chemistry, 20(8), 5782-5788.

Khan MR, Imran RM, Bukhari H (2008). Continuous proteolysis of casein by the cell bound protease of Carum copticum. Asian Journal of Chemistry, 20(7), 5729-5735.

Khan MR, Talib A (1986). Immobilization of Protease of Calotropis procera. Pakistan Journal of Scientific and Industrial Research, 2(4), 259-262.

Kilbanov AM (1983). Immobilized Enzymes and Cells as Practical Catalysts. Science, 219(4585), 722-727.

Krishnamoorthi S, Banerjee A, Roychoudhury A (2015). Immobilized enzyme technology: potentiality and prospects. Journal of Enzymology and Metabolism, l(1), 104.

Lee YC, Huh MH (1999). Development of a biosensor with immobilized D-amino acid oxidase for determination of L-amino acids. Journal of Food Biochemistry, 23, 173185.

Li S, Rosenzweig Z (1997). A fiber optic sensor for rapid analysis of bilirubin in serum. Analytica Chimica Acta, 353, 263-273.

Liang JF, Li YT, Yong VC (2000). Biomedical Application of Immobilized Enzymes. Journal of Pharmaceutical Sciences, 89(8), 979-990.

Liu DZ, Kai G, Kang C, Ni LH, Yao SZ (1997). A highly sensitive amperometric creatinine sensor. Analytica Chimica Acta, 351, 151-158.

Magnani M, Fazi A, Mangani F, Rossi L, Mancini U (1993). Methanoldetoxification byenzyme-loaded erythrocytes. Biotechnology and Applied Biochemistry, 18, 217-226.

Marazuela MD, Cuesta B, Moreno Bondi MC, Quejido A (1997). Free cholesterol fiber-optic biosensor for serum samples with simplex optimization. Biosensors and Bioelectronics, 12, 233-240.

Marazuela MD, Moreno-Bondi MC (1998). Determination of choline-containing phospholipids in serum with a fiber optic biosensor. Analytica ChimicaActa, 374, 19-29. 
Markoglou N, Wainer IW (2003). Immobilized enzyme reactors in liquid chromatography: On-line bioreactors for use in synthesis and drug discovery, Wilson ID (Ed.). In: Handbook of Analytical Separations, Elsevier Science B.V., Vol. 4, pp. 215-234.

Matosevic S, Szita N, Banganz F (2011). Fundamentals and applications of immobilized microfluidic enzymatic reactors. Journal of Chemical Technology and Biotechnology, 86(3),325-334.

Mintzer DM, Hauptman SP (1983). Lymphosarcoma cell leukemia and other non-Hodgkin's lymphomas in leukemic phase. American Journal of Medicine, 75(1), 110-120.

Mohamad NR, Marzuki NH, Buang NA, Huyop F, Wahab RA (2015). An overview of technologies for immobilization of enzymes and surface analysis techniques for immobilized enzymes. Biotechnology, Biotechnological Equipment, 29(2), 205-220.

Mori T, Tosa T, Chibata I (1974). Preparation and properties of asparaginase entrapped in the lattice of polyacrylamide gel. Cancer Research, 34(11), 30663068.

Nawaz SA, Khan MR (2000). Immobilization of the Proteases of Eurphorbia royleana. Pakistan Journal of Biological Sciences, 39(12), 2210-2212.
Rao KP (1995). Recent developments of collagen-based materials for medical applications and drug delivery systems. Journal of Biomaterials Science - Polymer Edition, 7(7), 623-645.

Shen Z, Cho W (1995). Highly efficient immobilization of phospholipase A2 and its biomedical applications. Journal of Lipid Research, 36, 1147-1151.

Tamer TM, Omer AM, Hassan M (2016). Methods of Enzyme Immobilization. International Journal of Current Pharmaceutical Review and Research, 7(6), 385-392.

Torres-Bacete J, Arroyo M, Torres-Guzmán R, de La Mata I, Castillón MP, Acebal C (2000). Optimization of 6aminopenicillanic acid (6-APA) production by using a new immobilized penicillin acylase. Biotechnology and Applied Biochemistry, 32(3), 173-177.

Wingard LB (1974). Developments and Challenge of Enzyme Engineering. In: Pye EK, Wingard LB. (Eds) Enzyme Engineering, Vol 2. Springer, Boston, MA.

\section{How to cite this article?}

Khan MR (2021). Current and future role of immobilized enzymes in medical field. Current Medical and Drug Research, 5 (1), Article ID 213. 